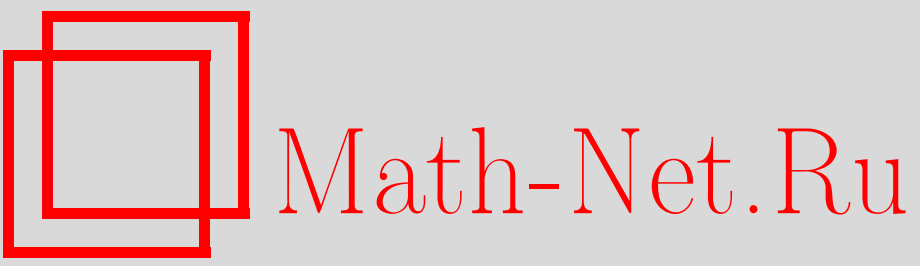

A. V. Baryshnikov, S. Yu. Katyshev, Схемы выработки общего ключа на основе линейных группоидов, Матем. вопр. криптогр., 2017, том 8, выпуск 1, 7-12

DOI: https://doi.org/10.4213/mvk211

Использование Общероссийского математического портала Math-Net.Ru подразумевает, что вы прочитали и согласны с пользовательским соглашением

http://www.mathnet.ru/rus/agreement

Параметры загрузки:

IP : 54.205 .225 .156

26 апреля 2023 г., 14:50:12 
МАТЕМАТИЧЕСКИЕ ВОПРОСЫ КРИПТОГРАФИИ

2017 T. 8 № 1 C. 7-12

УДК 519.719.2

Key agreement schemes based on linear groupoids ${ }^{1}$

\author{
A. V. Baryshnikov, S. Yu. Katyshev \\ Certification Research Center, LLC, Moscow
}

Получено 19.ІІІ.2016

\begin{abstract}
Authors present a study of the possibility to use special class of nonassociative groupoids (called linear) for the implementation of a key exchange protocol based on a generalization of Diffie-Hellmann algorithm. The necessity to use the power commutation and effective power calculation properties is proved. A specific example of linear groupoid over the elliptic curve is described.
\end{abstract}

Keywords: key exchange protocol, Diffie-Hellmann algorithm, non-associative groupoids, linear quasigroups, elliptic curves

\title{
Схемы выработки общего ключа на основе линейных группоидов
}

\section{А. В. Барышников, С. Ю. Катышев}

ООО «Центр сертификачионных исследований», Москва

Аннотация. Исследуется возможность использования одного класса неассоциативных группоидов (так называемых линейных группоидов) для реализации схемы выработки общего ключа, основанной на обобщении алгоритма Диффи - Хеллмана. Доказана необходимость использования коммутативности степеней и свойств эффективного вычисления степеней. Описан конкретный пример линейного группоида над группой точек эллиптической кривой.

Ключевые слова: протокол выработки общего ключа, алгоритм Диффи - Хеллмана, неассоциативные группоиды, линейные квазигруппы, эллиптические кривые.

Citation: Mathematical Aspects of Cryptography, 2017, v. 8, № 1, pp. 7-12 (Russian)

(c) Академия криптографии Российской Федерации, 2017 г.

\footnotetext{
${ }^{1}$ The article was submitted by the Organizing Committee of the Symposium CTCrypt'2016.
} 
We study the possibility to generalize the well-known Diffie-Hellmann algorithm [3] which implements a key exchange protocol by means of cyclic groups. We suggest to use a non-associative groupoid instead of a group. Let us introduce the corresponding definitions.

For an element $g$ of a finite groupoid $(\Omega, *)$ and given $r, l \in \mathbb{N}$ we define the right $r$-th powers and left $l$-th powers respectively by the equalities

$$
g^{[r]}=\underbrace{(\ldots((g * g) * g) \ldots)}_{r \text { factors }}, \quad{ }^{[l]} g=\underbrace{(\ldots(g *(g * g)) \ldots)}_{l \text { factors }} .
$$

We say that $g$ has commuting right powers, or that $g$ is a CRP-element, if

$$
\forall m, n \in \mathbb{N}: \quad g^{[m][n]}=g^{[n][m]} .
$$

If this identity is valid for any element $g \in \Omega$, then we say that $(\Omega, *)$ is a CRP-groupoid. Similarly, using the condition $\forall m, n \in \mathbb{N}: \quad{ }^{[m][n]} g={ }^{[n]}[m] g$ we define elements and groupoids with commuting left powers, or CLP-elements and CLP-groupoids, respectively.

Algorithm 1 of key exchange. After choosing a (non-secret) CRP-element $g$ of a groupoid $\Omega$, the users $A$ and $B$ independently choose arbitrary numbers $r_{A}, r_{B} \in \mathbb{N}$, and exchange the elements $g^{\left[r_{A}\right]}$ and $g^{\left[r_{B}\right]}$. Then they form the shared secret key $g^{\left[r_{A}\right]\left[r_{B}\right]}=g^{\left[r_{B}\right]\left[r_{A}\right]}$.

The complexity of revealing the secret key by an observer having access to the public information $g, g^{\left[r_{A}\right]}, g^{\left[r_{B}\right]}$ does not exceed the complexity of the right discrete logarithm in the groupoid, i. e. the complexity of solving the equation $g^{[x]}=h$.

A natural generalization of this approach to the construction of key exchange protocols is the combination of right and left powers.

We say that a groupoid $(\Omega, *)$ is a groupoid with commuting powers (CP-groupoid) if it is a CLP- and CRP-groupoid and also for any $g \in \Omega$ and any $l, r \in \mathbb{N}$ the following equality holds: ${ }^{[l]}\left(g^{[r]}\right)=\left({ }^{[l]} g\right)^{[r]}$.

We say that an element $g$ of any groupoid $(\Omega, *)$ is a CP-element if it generates a CP-groupoid.

Using a CP-element $g$ we can propose the following algorithm.

Algorithm 2 of a key exchange. Users $A$ and $B$ independently choose positive integers $r, m, s, n$ such that $r \leqslant m, s \leqslant n$ and sets of numbers $a_{1}, \ldots, a_{m} \in \mathbb{N}$ and $b_{1}, \ldots, b_{n} \in \mathbb{N}$. Then they exchange the messages

$$
K_{A}=\left[a_{1}\right] \ldots\left[a_{r}\right] g^{\left[a_{r+1}\right] \ldots\left[a_{m}\right]} \text { and } K_{B}={ }^{\left[b_{1}\right] \ldots\left[b_{s}\right]} g^{\left[b_{s+1}\right] \ldots\left[b_{n}\right]} \text {. }
$$


After that the users $A$ and $B$ calculate the shared secret key

$$
K_{A B}={ }^{\left[a_{1}\right] \ldots\left[a_{r}\right]} K_{B}^{\left[a_{r+1}\right] \ldots\left[a_{m}\right]}={ }^{\left[b_{1}\right] \ldots\left[b_{s}\right]} K_{A}^{\left[b_{s+1}\right] \ldots\left[b_{n}\right]} .
$$

It was shown in [1] that recovering of the shared secret key in Algorithm 2 is not harder than the generalized discrete logarithm problem, i. e. of finding some pair of positive integers $u, v$ and some sets $\left(x_{1}, \ldots, x_{v}\right), x_{i} \in \mathbb{N}, i \in \overline{1, v}$, satisfying the equation $\left[x_{1}\right] \ldots\left[x_{u}\right] g^{\left[x_{u+1}\right] \ldots\left[x_{v}\right]}=h$, if such numbers exist.

The paper [1] provides examples of nonassociative groupoids, that may be used in algorithms above.

Let $(\Omega, \cdot)$ be an arbitrary finite semigroup with identity element $e$. In what follows we will set $g^{0}=e$ for any $g \in \Omega$. Let $\left(\Omega^{\Omega}, \circ\right)$ be the semigroup of all mappings $\Omega \rightarrow \Omega$ and $\widetilde{\operatorname{Aut}}(\Omega)=\widetilde{\operatorname{Aut}}(\Omega, \cdot)$ be the subgroup of the semigroup $\left(\Omega^{\Omega}, \circ\right)$ consisting of all automorphisms and anti-automorphisms of the semigroup $(\Omega, \cdot)$.

Fixing two commuting (anti-)automorphisms $\sigma, \tau \in \widetilde{\operatorname{Aut}}(\Omega), \quad \sigma \tau=\tau \sigma$, we define a new operation on $\Omega$ by the following condition:

$$
\forall x, y \in \Omega \quad x * y=\sigma(x) \cdot \tau(y) .
$$

So we obtain a groupoid $(\Omega, *)$ which is a quasigroup if and only if $(\Omega, \cdot)$ is a group. Such quasigroups form a class of medial quasigroups [2].

If $(\Omega, \cdot)$ is an Abelian semigroup, then we will call $(\Omega, *)$ a medial groupoid.

Theorem 1. If $(\Omega, \cdot)$ is an Abelian semigroup and $\sigma, \tau$ are its commuting (anti-) automorphisms then the groupoid $(\Omega, *)$ with operation (3) is a CP-groupoid.

In [1] the power commutation property for medial quasigroups was proved, as well as possibility to implement effectively public key distribution algorithms based on this quasigroups (there exist fast exponentiation methods, in particular, a generalization of binary exponentiation algorithm). As a result of analysis of the above algorithms an upper estimate for the complexity of discrete logarithm problem was obtained. In this paper we present a generalisation of the medial groupoids, namely linear groupoids.

Let $(\Omega, \cdot)$ be an arbitrary finite semigroup with identity element $e$. Fixing two commuting (anti-)automorphisms $\sigma, \tau \in \widehat{\operatorname{Aut}}(\Omega), \quad \sigma \tau=\tau \sigma$, and an element $h \in \Omega$ we define a new operation on $\Omega$ by the condition

$$
\forall x, y \in \Omega \quad x *_{h} y=\sigma(x) \cdot \tau(y) \cdot h .
$$

Similarly we obtain a groupoid $\left(\Omega, *_{h}\right)$, which is a quasigroup if and only if $(\Omega, \cdot)$ is a group. We will call $\left(\Omega, *_{h}\right)$ a linear groupoid. 
For the linear groupoids a power commutation property is proved.

Theorem 2. If $(\Omega, \cdot)$ is an Abelian semigroup, $\sigma, \tau$ are its commuting (anti-) automorphisms and $h$ is an arbitrary element of $\Omega$, then the groupoid $\left(\Omega, *_{h}\right)$ with operation (4) is a CP-groupoid.

Let $(\Omega, \star)$ be a groupoid with the operation

$$
\forall x, y \in \Omega: \quad x \star y=\sigma(x) \cdot y .
$$

For any $\alpha \in \Omega$ let $\alpha^{\left[{ }_{h} n\right]}$ and $\alpha^{[\star n]}$ denote right $n$-th powers of the element $\alpha$, respectively in $\left(\Omega, *_{h}\right)$ and in $(\Omega, \star)$. Note that $\alpha^{[\star n]}=\sigma^{n-1}(\alpha) \cdot \ldots \cdot \sigma(\alpha) \cdot \alpha$.

If $(\Omega, *)$ is a linear groupoid then even if $\tau \neq \varepsilon$, the calculation of powers and logarithms in this groupoid may be reduced to the solution of corresponding problems in a groupoid with an operation of the form (5).

Theorem 3. Let $(\Omega, \cdot)$ be a group with identity element e. If $\left(\Omega, *_{h}\right)$ is the linear groupoid with the operation (4), then for any $n \in \mathbb{N}$ the operation (5) satisfies the equation $g^{\left[{ }_{h} n\right]}=\alpha^{[\star n-1]} \cdot g$, where $\alpha=\sigma(g) \tau(g) h g^{-1}$.

This theorem allows us to use algorithms from [1] for the calculation of the $n$-th power in linear groupoid $\left(\Omega, *_{h}\right)$ with complexity $O\left(A U T(\sigma, n) \log _{2} n\right)$ operations in the group $(\Omega, \cdot)$, where $\operatorname{AUT}(\sigma, n)$ is the complexity of calculation of $\sigma^{n}(a)$ for an arbitrary element $a$ of the group $(\Omega, \cdot)$.

If automorphisms are defined by their actions on generating elements, then the upper estimate takes the form $O\left(\log ^{2}|\Omega|\right)$. If the automorphisms $\sigma, \tau$ have small orders (as compared with the cardinality of the group), this value is equal to the complexity estimate of Diffie-Hellmann protocol realization. The complexity of the left power calculation is estimated similarly.

Let $(\Omega, *)$ be an arbitrary CP-groupoid. We say that a map $\delta$ of the groupoid $(\Omega, *)$ onto itself imitates a secret key $\left(r, n, a_{1}, \ldots, a_{m}\right)$ of user $A$ if for any secret key $\left(s, n, b_{1}, \ldots, b_{n}\right)$ of user $B$ the following equality holds:

$$
\delta\left(\left[b_{1}\right] \ldots\left[b_{s}\right] g^{\left[b_{s+1}\right] \ldots\left[b_{n}\right]}\right)=\left[a_{1}\right] \ldots\left[a_{r}\right]\left[b_{1}\right] \ldots\left[b_{s}\right] g^{\left[b_{s+1}\right] \ldots\left[b_{n}\right]\left[a_{r+1}\right] \ldots\left[a_{m}\right]},
$$

i.e. if using the map $\delta$ instead of the map defined by the key of user $A$, we nevertheless get the true value of the common key. Shortly we will call $\delta$ an imitating map.

The map $\psi: \psi(g)=\left[a_{1}\right] \ldots\left[a_{r}\right] g^{\left[a_{r+1}\right] \ldots\left[a_{m}\right]}$ is a homomorphism if $h=e$, the neutral element of the group $(G, \cdot)$. 
Theorem 4. Let $(\Omega, \cdot)$ be an Abelian group, $\left(\Omega, *_{h}\right)$ be linear quasigroup, $\delta$ be a homomorphism of the group $(\Omega, \cdot)$ that commutes with automorphisms $\sigma$ and $\tau$. Then $\delta$ is a homomorphism of the $\left(\Omega, *_{h}\right)$ if and only if $\delta(h)=h$.

Finding a shared secret key is still possible by means of solving the discrete logarithm problem. To obtain upper bounds for the key exchange protocols based on non-associative groupoids, a generalisation of well-known Hellman's method was constructed.

Let $(\Omega, *)$ be a CP-groupoid, $K=\left\{\left(u, v, a_{1}, \ldots, a_{v}\right) \mid u, v, a_{i} \in \mathbb{N}, i \in \overline{1, v}\right\}$ be a set of powers (set of secret keys in algorithm 2). On this set we define the relation $\varepsilon$ by the rule

$\left(u, v, a_{1}, \ldots, a_{v}\right) \stackrel{\varepsilon}{\sim}\left(u^{\prime}, v^{\prime}, a_{1}^{\prime}, \ldots, a_{v}^{\prime}\right) \Leftrightarrow{ }^{\left[a_{1}\right] \ldots\left[a_{u}\right]} g^{\left[a_{u+1}\right] \ldots\left[a_{v}\right]}={ }^{\left[a_{1}^{\prime}\right] \ldots\left[a_{u^{\prime}}^{\prime}\right]} g^{\left[a_{u^{\prime}+1}\right] \ldots\left[a_{v}^{\prime}\right]}$.

It is easy to check that relation $\varepsilon$ is an equivalence relation on the set $K$ and, hence, the set $K$ is divided into equivalence classes. Denote

$$
K_{\varepsilon}=\frac{|K|}{[\vec{x}]_{\varepsilon}}
$$

where $[\vec{x}]_{\varepsilon}$ is an equivalence class containing element $\vec{x}$.

Theorem 5. Considered algorithm based on Hellman's method allows to find a shared secret key with complexity $K_{\varepsilon}^{2 / 3}$.

Note that if all equivalence classes have the same cardinality, then the value $K_{\varepsilon}$ does not exceed cardinality of CP-groupoid $\Omega$. Thus finding a shared secret key requires $O\left(|\Omega|^{2 / 3}\right)$ memory cells and in the average $O\left(|\Omega|^{2 / 3}\right)$ operations at the operational stage.

As an example of algebraic support for algorithms 1 and 2, one can use a linear quasigroup over elliptic curve. Consider a finite field $F_{q}$ of $q$ elements with char $F_{q}>3$ and its algebraic extension $F_{q^{m}}$. Define an elliptic curve $(E, \oplus)$ over the field $F_{q^{m}}$ by the equation $y^{2}=x^{3}+a x+b, a, b \in F_{q}$. Define the operation $*_{h}$ over $E$ by the equation $\forall P, S \in E: P *_{h} S=\sigma(P) \oplus S \oplus h$, where $\sigma$ is a Frobenius endomorphism, that operates on the arbitrary point $P=(x, y)$ of elliptic curve $E\left(F_{q^{m}}\right)$ by the following way: $\sigma(x, y)=\left(x^{q}, y^{q}\right)$. Thus $\left(E, *_{h}\right)$ is a linear quasigroup.

Choose $P \in(E, \oplus)$ to be an element of large order, such that $\sigma(P) \notin\langle P\rangle$. We propose the following algorithm for key exchange.

User $A$ chooses a pair of numbers $\left(l_{A}, r_{A}\right), l_{A}, r_{A} \in \mathbb{N}$, and $B$ independently chooses $\left(l_{B}, r_{B}\right), l_{B}, r_{B} \in \mathbb{N}$. Then they exchange the messages $K_{A}={ }^{\left[l_{A}\right]} P^{\left[r_{A}\right]}$ and $K_{B}={ }^{\left[l_{B}\right]} P^{\left[r_{B}\right]}$. After that the users $A$ and $B$ calculate the shared secret key $K_{A B}:{ }^{\left[l_{A}\right]}\left(\left[l_{B}\right] P^{\left[r_{B}\right]}\right)^{\left[r_{A}\right]}={ }^{\left[l_{B}\right]}\left({ }^{\left[l_{A}\right]} P^{\left[r_{A}\right]}\right)^{\left[r_{B}\right]}$. 
For linear groupoids theoretically justified power commutation required for their use in algorithms 1 and 2. Obtained results allow to use described in the article [1] fast (polynomial) algorithms for exponentiation in the case of linear groupoid. This makes it possible to implement presented algorithms with the same complexity as in the classic Diffie-Hellman protocol. Moreover, these algorithms may be constructed over elliptic curves, that confirmed the possibility of their efficient implementation on a modern hardware.

\section{Список литературы}

[1] Katyshev S. Yu., Markov V. T., Nechaev A. A., "Application of non-associative groupoids to the realization of an open key distribution procedure”, Дискретная математика, 26:3 (2014), 45-64 (in Russian); Engl. transl., Discrete Math. Appl., 25 :1 (2015), 9-24.

[2] Belyavskaya G.B., Tabarov A. Kh., "Identities with permutations leading to linearity of quasigroups”, Дискретная математика, $21: 1$ (2009), 36-51 (in Russian); Engl. transl., Discrete Math. Appl., 19:2 (2009), 173-190.

[3] Diffie W., Hellman M.E., "New directions in cryptography", IEEE Trans. Inf. Theory, $22: 6$ (1976), 644-654.

[4] Hellman M., “A cryptanalytic time-memory trade-off”, IEEE Trans. Inf. Theory, 26 :4 (1980), 401-406.

[5] Silverman J., The Arithmetic of the Elliptic Curves, Heidelberg etc.: Springer, 1986. 